\title{
Determining the point of subjective ambiguity of ambiguous biological-motion figures with perspective cues
}

\author{
Ben Schouten and Karl Verfaillite \\ University of Leuven, Leuven, Belgium
}

\begin{abstract}
Orthographic frontal/back projections of biological-motion figures are bistable: The point-light figure in principle can be perceived either as facing toward the viewer or as facing away from the viewer. Some point-light actions - for example, walking - elicit a strong "facing bias": Despite the absence of objective cues to depth, observers tend to interpret the figure as facing toward the viewer in most of the cases. In this article, we present and experimentally validate a technique that affords full experimental control of the perceived in-depth orientation of point-light figures. We demonstrate that by parametrically manipulating the amount of perspective information in the stimulus, it is possible to obtain any desired level of subjective ambiguity. Directions for future research, in which this technique can be fruitfully implemented, are suggested. Program code of a demo is provided that can be modified easily for program code of new experiments. The demo and QuickTime movie files illustrating our perspective manipulation technique may be downloaded from http://brm.psychonomic-journals.org/ content/supplemental.
\end{abstract}

Gunnar Johansson (1973) was the first to introduce the point-light method to investigate the perception of biological motion. He attached light-emitting bulbs or lightreflecting patches to the bodies of moving actors and filmed them under such conditions that only the lights were perceptible for observers. Ever since, this pointlight technique has been widely used and refined by vision scientists studying human action perception. During the past decades, the research on biological motion has demonstrated the richness of information contained in these so-called point-light figures. Despite the strong degradation of visual information, observers are able to effortlessly extract a variety of behaviorally relevant information, such as the type of action (Dittrich, 1993; Vanrie \& Verfaillie, 2004), the identity of the actor (Cutting \& Kozlowski, 1977; Loula, Prasad, Harber, \& Shiffrar, 2005), their gender (Kozlowski \& Cutting, 1977; Pollick, Kay, Heim, \& Stringer, 2005; Troje, 2002), emotional state (Dittrich, Troscianko, Lea, \& Morgan, 1996; Montepare, Goldstein, \& Clausen, 1987; Pollick, Paterson, Bruderlin, \& Sanford, 2001), and age (Montepare \& ZebrowitzMcArthur, 1988). See Verfaillie (2000) for some notes on the early history of this line of research.

Only recently, however, has the in-depth perception of point-light figures been systematically investigated. Vanrie, Dekeyser, and Verfaillie (2004) presented observers with an orthographic 2-D projection of a symmetrical point-light walker derived from the captured 3-D coordinates of a walking human (for details, see Dekeyser, Ver- faillie, \& Vanrie, 2002). The stimulus is bistable, in the sense that it has two veridical and anatomically plausible interpretations of a human figure: The figure is either perceived to be "facing the viewer" (FTV) or it is perceived to be "facing away" (FA) from the viewer (see Figure 1). More importantly, Vanrie et al. demonstrated that, despite the theoretically ambiguous nature of this stimulus, on average, people tend to interpret the walker as facing toward the observer in more than $80 \%$ of the cases.

Subsequent research on the in-depth perception of point-light figures (Vanrie \& Verfaillie, 2006) demonstrated perceptual bistability for other point-light actions (Vanrie \& Verfaillie, 2004) but also revealed that the presence of a preferred interpretation strongly depends on the performed action, suggesting that the process of depth assignment takes into account the movements of the pointlight figure.

To date, it is still not clear why observers exhibit such a strong tendency to interpret some point-light actions as facing toward the viewer. A growing group of researchers is now studying contextual or stimulus-specific factors such as the gender of the point-light figures (Brooks et al., 2008), looming and receding sounds (Mendonça \& Santos, 2008; Schouten, Moyens, Brooks, van der Zwan, \& Verfaillie, 2008), or the presence of more than one pointlight figure (Vanrie \& Verfaillie, 2008), or the presence of more than one point-light figure (Vanrie \& Verfaillie, 2008), that seem to affect the perceived in-depth orientation of point-light figures. 


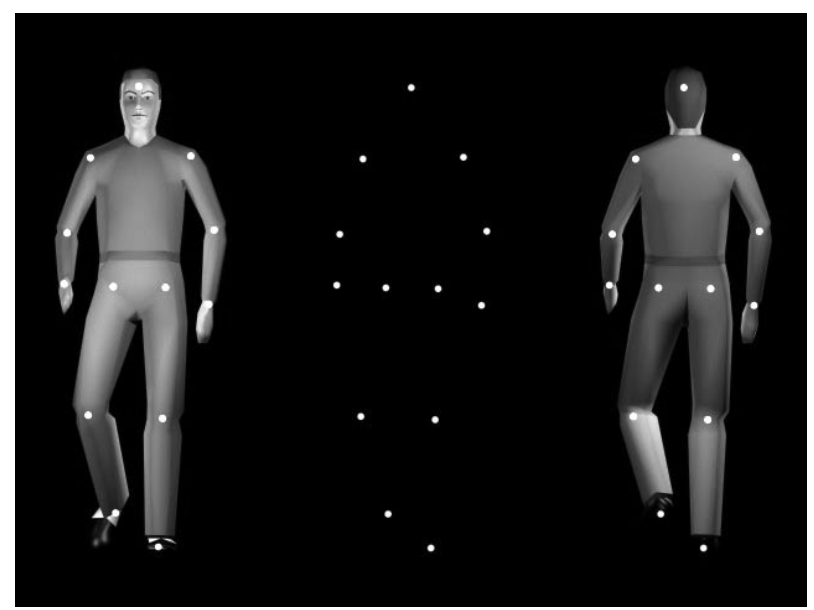

Figure 1. Illustration of a single frame of the ambiguous pointlight walker (middle) and the two veridical interpretations with the point lights superimposed. Although both veridical interpretations are equally likely, observers prefer the "facing the viewer" interpretation (left). Figure adapted from "Bistability and Biasing Effects in the Perception of an Ambiguous Point-Light Walker," by J. Vanrie, M. Dekeyser, and K. Verfaillie, 2004, Perception, 33, p. 548. Copyright 2004 by Pion. Adapted with permission.

Studying factors that affect the in-depth orientation perception of point-light figures, however, is often complicated by the relatively large interindividual variation in the average perceived in-depth orientation. For example, when repeatedly confronted with the same point-light figure, some observers might judge the in-depth orientation of the point-light figure on average as rather ambiguous, whereas for other observers the figure is on average judged to be convincingly facing the viewer. The relatively large variation between observers can cause a decrease in power of the test when studying the effect of a contextual variable across observers, because for at least a part of the observers, the effect of the contextual variable will result in a ceiling or floor effect. Hence, to more meaningfully compare the effect of a contextual variable on the perceived in-depth orientation across observers, it would be convenient if the subjective experiences could be aligned across observers to serve as a baseline or control condition. The ideal baseline to prevent ceiling and floor effects would be the point where the figure in half of the cases is perceived to be oriented toward the observer and in the other half of the cases is perceived to be oriented away from the observer. At this point, the in-depth orientation of the point-light figure is, for a particular observer, subjectively ambiguous. Analogous to other measures of subjective equality (PSE; e.g., Ernst \& Banks, 2002) or subjective simultaneity (PSS; e.g., Arrighi, Alais, \& Burr, 2006), we refer to this point as the point of subjective ambiguity (PSA).

To psychophysically determine the PSA for a particular observer, the first prerequisite is to identify a physical variable that reliably affects the perceived in-depth orientation of a point-light figure. One such variable is the amount of perspective information that is conveyed by the figure. Indeed, Vanrie et al. (2004) showed that the perceived in-depth orientation can be altered by introducing perspective cues. However, in their experiment, the perspective manipulation was only categorical. That is, Vanrie et al. showed that by using a (strong) perspective projection instead of an orthographic projection, the facing bias can be countered. The perspective projection of a point-light figure that is oriented away from the viewer elicited the in-depth orientation interpretation consistent with the figure's veridical orientation. The second prerequisite to psychophysically determine the PSA, however, is that the physical variable can be parametrically controlled and that parametrical variations of the variable lead to gradual variations in the perceived in-depth orientation. The goal of the present article is to verify whether, indeed, parametrically manipulating the amount of perspective information in a point-light figure can provide a way to (1) gradually manipulate the perceived in-depth orientation and (2) determine the PSA for each observer individually.

In this article, we first present a technique that offers complete experimental control of the perceived in-depth orientation of a point-light stimulus by parametrically manipulating the amount of available perspective information. Second, we experimentally validate the technique. Finally, we discuss potential future research and provide the program code of a demo that can serve as a helpful starting point in programming future experiments.

\section{Technique}

Generally, observers are unaware of the fact that what they visually perceive is actually the result of a reconstruction of the 3-D world projected on the 2-D retinal surface. Although there are various ways to graphically project 3-D objects to a 2-D surface, two types of projections are specifically relevant to the present work: orthographic projection and perspective projection.

Consider a human 3-D figure in a 3-D space that is only represented by a collection of small spheres positioned in the middle of the major joints (shoulders, elbows, wrists, hips, knees, and ankles) and the head. Consider also the projection of this 3-D figure on a 2-D surface (e.g., a computer screen or a piece of paper), parallel to its coronal plane. In an orthographic projection, in which all projection lines are parallel, any information regarding the distance between the 3-D figure and the projection plane is effectively removed. Hence, changing the location of the spheres in depth will not change their location on the projection plane in any way. This projection effectively removes all perspective cues to depth (see Movie 1, in the supplement for this article). In a perspective projection, on the contrary, projection lines are converging at one point (the vanishing point). Hence, changing the distance between the collection of spheres and the projection plane will change the size, shape, and location of the 2-D projection of the spheres on the projection plane. This projection does contain perspective cues to depth (see Movie 2).

In their study, Vanrie et al. (2004) applied the perspective projection to a 3-D point-light figure as a categorical 
manipulation. That is, the perspective projection consisted of only one particular observer-source distance with a fixed field-of-view angle. Because their main goal was to examine whether a strong global cue would render the true orientation of the figure more perceptible, they deliberately chose a projection distance that yielded a relatively strong perturbation of the point-light configuration. By doing so, they were able to successfully disambiguate the figure. Notably, Vanrie et al. referred to the perspective projection as an important tool to render the true orientation of the point-light figure more visible. The main rationale of the present study was to examine whether it would be possible to gradually manipulate the amount of perspective information present in a point-light walker to further exploit the usefulness of this tool.

To gradually vary the available perspective information in a point-light walker, in a perspective projection, the distance between the 3-D figure and the projection plane was parametrically manipulated. When the distance between the 3-D figure and the projection plane is decreased, the position, size, and shape distortions of the projection of the spheres on the projection plane increase. These distortions are in fact perspective cues. So, gradually boosting these distortions by further decreasing the distance between the 3-D figure and the projection plane gradually exaggerates the perspective cues.

As a result of decreasing the distance (D) between the 3-D figure and the projection plane, however, the size of the projected image (S) and hence, also, the overall size of the projected point-light figure increases. To overcome this side effect, it is sufficient to proportionally increase the fieldof-view angle $(\mathrm{V})$ of the perspective projection according to the following formula: $\mathrm{V}=2 * \arctan [\mathrm{S} /(2 * \mathrm{D})]$. This technique to incorporate perspective cues to depth is, among visual artists, also known as foreshortening. Movie 3 illustrates how the 2-D projection of the point-light figure varies as a result of the manipulation explained above.

\section{The Present Experiment}

Crucial for the applicability of the technique is that the parametric stimulus manipulations also lead to gradual and meaningful changes in the perceived orientation of a biological-motion figure. To test this, we conducted an experiment in which we parametrically increased the perspective information of a point-light walker who was oriented away from the observer. By measuring the proportion of FTV responses to a walker who was actually walking away, as a function of the amount of perspective information, we were able to verify whether the tendency to perceive the walker as facing the viewer (the facing bias under orthographic projection) could be gradually decreased and altered to the interpretation that matches its veridical in-depth orientation.

\section{METHOD}

\section{Participants}

Nine psychology students from the University of Leuven participated for course credit. All of the observers had normal or correctedto-normal vision and were naive to the aims of the experiment.

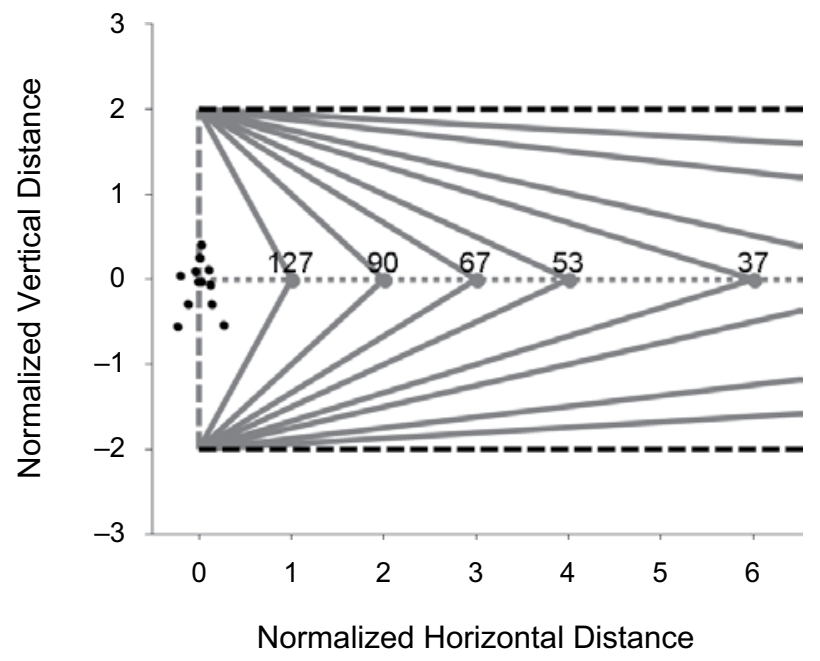

Figure 2. Schematic illustration of the lateral 2-D view of the 3-D projection space. The horizontal and vertical distances are normalized to the height of the point-light walker. The horizontal black dashed lines represent the projection lines of the orthographic projection. The solid gray lines represent the projection lines of the perspective projections. The black dots represent the point-light walker. The vertical gray dashed line indicates the size of the projected image (S). The gray dots illustrate the distance (D) between the projection plane and the point-light figure (only $6,4,3,2$, and 1 are shown). The angles that are formed between the gray lines at each gray dot illustrate the field-of-view angles (only $37^{\circ}, 5^{\circ}, 67^{\circ}, 90^{\circ}$, and $127^{\circ}$ are shown). The shorter the distance $D$, the larger the field-of-view angle is.

\section{Stimuli}

All point-light figures were derived from the same 3-D coordinates of a human walker, from which the translational component was removed (Dekeyser et al., 2002). As a result, the walker moved as if walking on a treadmill, in the center of the screen. The walker was facing away from the viewer. The perspective cues carrying the information concerning the veridical orientation of the point-light figure were gradually manipulated (nine levels) from absent (orthographic projection) to very strong. The nine levels of perspective information were composed of one orthographic projection (dashed green line in the left panel of Movie 3 and horizontal gray dashed line in Figure 2) and eight distance (D) manipulations (blue dots in the left panel of Movie 3 and gray dots in Figure 2) of the convergence point, corresponding to $32,16,8,6,4,3,2$, and 1 times the height of the walker. This resulted respectively in field-of-view angles of $7^{\circ}$, $14^{\circ}, 28^{\circ}, 37^{\circ}, 53^{\circ}, 67^{\circ}, 90^{\circ}$, and $127^{\circ}$ (see Movie 3 and Figure 2, angle between full lines). The height of the point-light figures (adjusted according to the viewing distance, as discussed above) subtended about $7^{\circ}$ of visual angle. Hence, the height of the projected image (screen height, $4 *$ height of the walker) subtended about $28^{\circ}$. Each dot subtended about 15 arc mins. Each stimulus presentation lasted about $4.5 \mathrm{sec}$ and consisted of three walking cycles, each consisting of two steps. The start position of the animation cycle was randomized across trials.

\section{Procedure}

Observers were seated in a dimly lit and sound-attenuated room. The observers' heads were immobilized by means of a chinrest. Viewing distance was $57 \mathrm{~cm}$ from a CRT monitor (refresh rate = $85 \mathrm{~Hz}$ ). On each trial, observers had to indicate by a keypress (arrow down for FTV, arrow up for FA) whether the visually presented stimulus was perceived as oriented toward or away from them. They were instructed to respond according to their own subjective experience 

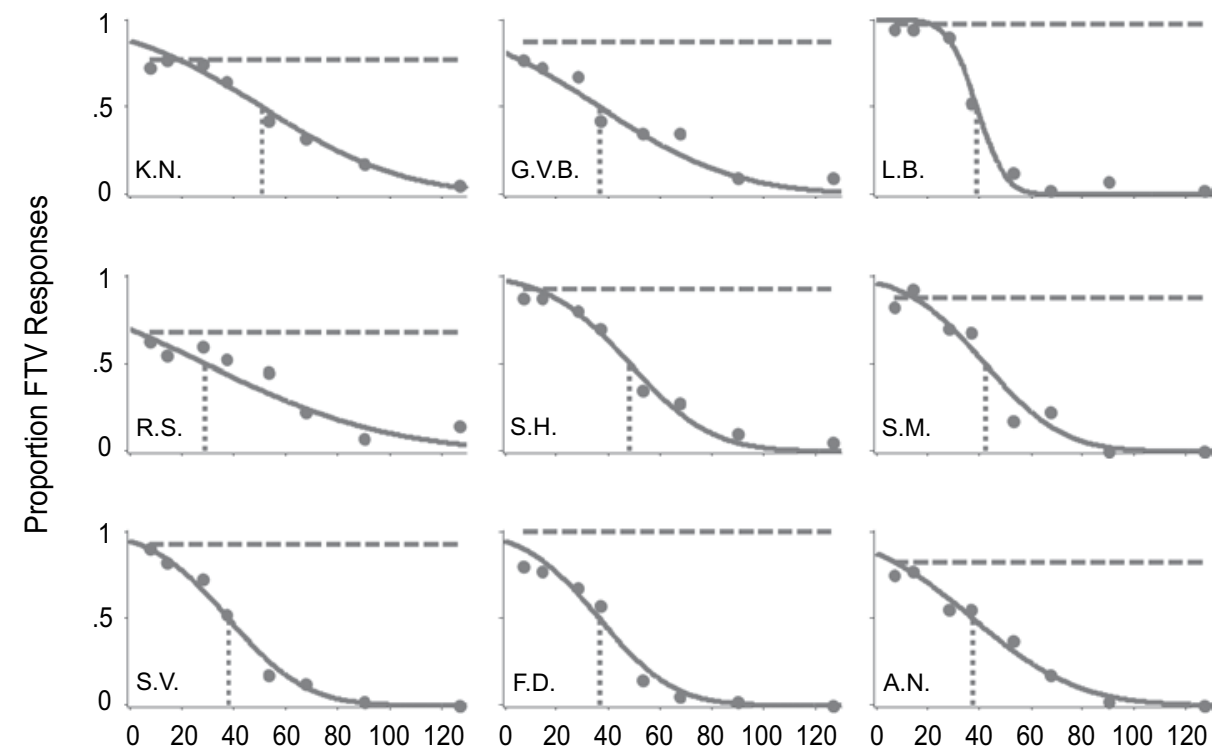

Field-of-View Angle

Figure 3. Proportions of "facing the viewer" (FTV) responses as a function of field-of-view angle for 9 observers. The horizontal dashed line represents the proportion of FTV responses when no cues to depth are present (orthographic projection). Filled dots represent the proportions of FTV responses for all perspective projections, and the full curved line represents their cumulative Gaussian fit. The vertical dotted line represents the point of subjective ambiguity (PSA), corresponding to $50 \%$ FTV responses.

and it was stressed that an equal distribution of both response alternatives was not necessary. In total, each observer completed 360 (9 levels $* 40$ repetitions) trials

\section{RESULTS}

Figure 3 plots the proportions of FTV responses for the orthographic projection, the proportions of FTV responses as a function of the field-of-view angle for the perspective projection, and the PSA for each of the 9 observers. Inspection of the figure points to two important observations. First, when the point-light walker is projected orthographically - that is, when no perspective cues are present-all observers are biased to interpret the figure as facing toward the viewer (horizontal dashed line), in line with the effect reported by Vanrie et al. (2004). This is confirmed by the high proportion of FTV responses for each observer $($ mean $=0.87 ; \min =0.68 ; \max =1.0)$ in the orthographic condition. Second, proportions of FTV responses gradually drop as more perspective cues are added to the figure walking away. Moreover, proportions of FTV responses as a function of field-of-view angle are well fit with a cumulative Gaussian function, as confirmed by the goodness-of-fit test on each individual data set: Deviance of the observations to the cumulative Gaussian fit (Wichmann \& Hill, 2001) was not significantly higher than simulated deviance (10,000 Monte Carlo simulations) for 8 out of 9 observers (all $p$ values $>.05$, except for observer S.M., $p=.01$ ). PSA values of the 9 observers, expressed as the field-of-view angle, vary between $29^{\circ}$ (R.S.) and $51^{\circ}$ (K.N.), with a mean of $40^{\circ}(S D=6.64)$.
Slopes vary between -0.011 and -0.080 , with a mean of $-0.023(S D=0.022)$. Note that the choice to express the ordinate values as the field-of-view angle of the perspective projection is somewhat arbitrary. Proportions of FTV responses can be just as well plotted and fitted as a function of, for example, the distance D or the logarithm of the distance $\mathrm{D}$.

\section{DISCUSSION}

First, earlier findings demonstrating that orthographically projected point-light figures elicit a strong facing bias are confirmed (Vanrie et al., 2004). Second, the results indicate that the technique presented here is well suited for gradually manipulating the perceived in-depth orientation of a bistable biological-motion figure: The perceived in-depth orientation of a point-light walker who is oriented away from the viewer gradually changes from the FTV interpretation (facing bias) to the veridical FA interpretation when perspective cues are introduced parametrically. Proportions of FTV responses as a function of added perspective cues are, at the individual observer level, well fit with a cumulative Gaussian psychometric function. The single significant deviance for the fit to the data of observer S.M. indicates overdispersion (unexplained variability), presumably provoked by unstable behavior in the task.

In our experiment, the mean of the cumulative Gaussian function indicates the PSA, the stimulus of which, the perceived in-depth orientation, is subjectively perfectly ambiguous. This 50\% threshold gives an estimation of 
the amount of perspective that a particular observer, or a group of observers, needs to counter the facing bias. Moreover, the PSA can be a good reference point in studying factors affecting the perceived in-depth orientation. By comparing the proportions of FTV responses of the subjectively ambiguous point-light walker with proportions of FTV responses to the same ambiguous walker but with added contextual cues, the disambiguating effect of the contextual cues can be directly measured as an increase or decrease in the proportion of FTV responses. Taking the individual PSA as a baseline for each observer gives the opportunity to standardize subjective experiences across observers and to prevent ceiling and floor effects.

To further illustrate the potential usefulness of the experimental tool presented here, we briefly discuss two lines of research we are currently pursuing. First, Brooks et al. (2008) recently reported a striking correlation between the depicted gender and the perceived in-depth orientation of a bistable biological-motion walker: Male walkers generally were perceived as approaching the observer, whereas female walkers were seen as retreating. To examine the effect in more detail, a technique similar to the tool presented here was used: Perspective cues disambiguating the walker's facing direction were gradually added to the figure judged as most female, to the figure judged as most male, and to a gender-neutral walker. As expected, perspective cues affected facing judgments, but much stronger cues signaling a receding conspecific had to be added to the neutral and male walker than to the female walker, in order to change the "facing bias" for the male and neutral walker into a "retreating bias." Schouten, Troje, Brooks, van der Zwan, and Verfaillie (2010) have reported on a follow-up study that examines both the generalizability of the observation and the effect of observer sex. Second, the technique offers opportunities to study cross-modal perception of actions. There is indeed evidence that mammalian observers represent actions in an audiovisual format (e.g., Barraclough, Xiao, Baker, Oram, \& Perrett, 2005; Bidet-Caulet, Voisin, Bertrand, \& Fonlupt, 2005; Keysers et al., 2003; Kohler et al., 2002), rather than in separate visual and auditory codes that are merged only in a relatively late processing stage. Research on audiovisual integration has suggested that auditory effects on visual perception are strongest when the visual information is weak or ambiguous - also known as the rule of inverse effectiveness (Meredith \& Stein, 1983; but see Holmes, 2007). Hence, when the visual information concerning the in-depth orientation of a point-light walker is maximally ambiguous, unambiguous auditory looming or receding information might affect the perceived indepth orientation most strongly. Our technique could be a useful tool to examine this issue. Pilot psychophysical experiments (Mendonça \& Santos, 2008; Schouten et al., 2008) indeed suggest that looming and receding sounds can affect the perceived in-depth orientation of a pointlight walker. More research is necessary to test at what processing level looming and receding sounds can affect the perceived in-depth orientation of a multistable pointlight figure.
Besides pointing to the advantages and the possibilities offered by this technique, we also want to be clear on its limitations. First, because the technique is based on an exaggeration of the distortions related to changes in depth, it probably will be less efficient for point-light actions in which all joints/dots remain in the same depth plane (orthogonal to the observer's line of sight). For example, a frontal/back view of a point-light jumper in which the arms and legs only move sideways in the figure's coronal plane would not be an appropriate stimulus for our technique because it contains only a small amount of potential perspective information. Interestingly, the presence or absence of a facing bias to a frontal/back view indeed seems to be related to the amount of motion in the sagittal plane: Actions that do not contain substantial motion in the sagittal plane generally do not elicit a facing bias (Vanrie \& Verfaillie, 2006). Second, the distortions at the projection plane include distortions of the shape, size, and position of the dots that may function as several local cues instead of one global cue. The magnitude of the distortion increases proportionally with the amplitude of the horizontal, vertical, and depth displacement of the dot relative to the center of the figure. It is not yet clear to what extent these individual distortions contribute to the overall change in perceived orientation. Therefore, it would be interesting to test to what extent isolated shape, size, or position changes lead to changes in the perceived in-depth orientation.

To support future research, in the Appendix we provide the code of a demo that can serve as an easily modifiable starting point for new experiment code.

\section{CONCLUSIONS}

The technique presented here enables the study of factors that affect the perceived in-depth orientation over the entire range of possible depth interpretations (from convincingly facing the viewer to convincingly facing away from the viewer). More specifically, the technique makes it possible to express factors affecting the in-depth interpretation quantitatively, as changes in the mean (PSA) and slope of a psychometric curve. We are convinced that a systematic experimental study of factors affecting the perceived in-depth orientation will shed light on the processes of depth assignment in point-light figures and will help to identify the causal factors in the intriguing and robust bias to interpret some point-light figures as facing toward the observer. We hope that the tool provided in this article will contribute to this research.

\section{AUTHOR NOTE}

This research was supported by the Scientific Research Fund-Flanders, FWO G.0621.07, supporting the study of audiovisual perception of biological motion. We thank Sarah Moens for her help with the data collection. Correspondence concerning this article should be addressed to B. Schouten, Tiensestraat 102, 3000 Leuven, Belgium (e-mail: ben .schouten@psy.kuleuven.be).

\section{REFERENCES}

Arrighi, R., Alais, D., \& Burr, D. (2006). Perceptual synchrony of au- 
diovisual streams for natural and artificial motion sequences. Journal of Vision, 6(3, Art. 6), 260-268. doi:10.1167/6.3.6

Barraclough, N. E., Xiao, D., Baker, C. I., Oram, M. W., \& PerRETT, D. I. (2005). Integration of visual and auditory information by STS neurons responsive to the sight of actions. Journal of Cognitive Neuroscience, 17, 377-391. doi:10.1162/0898929053279586

Bidet-Caulet, A., Voisin, J., Bertrand, O., \& Fonlupt, P. (2005). Listening to a walking human activates the temporal biological motion area. NeuroImage, 28, 132-139. doi:10.1016/j.neuroimage .2005 .06 .018

Brooks, A., Schouten, B., Troje, N. F., Verfaillie, K., Blanke, O., \& VAN DER ZWAN, R. (2008). Correlated changes in perceptions of the gender and orientation of ambiguous biological motion figures. Current Biology, 18, 728-729. doi:10.1016/j.cub.2008.06.054

Cutting, J. E., \& Kozlowski, L. T. (1977). Recognizing friends by their walk: Gait perception without familiarity cues. Bulletin of the Psychonomic Society, 9, 353-356.

Dekeyser, M., Verfaillie, K., \& Vanrie, J. (2002). Creating stimuli for the study of biological-motion perception. Behavior Research Methods, Instruments, \& Computers, 34, 375-382.

DitTRICH, W. H. (1993). Action categories and the perception of biological motion. Perception, 22, 15-22. doi:10.1068/p220015

Dittrich, W. H., Troscianko, T., Lea, S. E. G., \& Morgan, D. (1996). Perception of emotion from dynamic point-light displays represented in dance. Perception, 25, 727-738. doi:10.1068/p250727

ERnST, M. O., \& BANKS, M. S. (2002). Humans integrate visual and haptic information in a statistically optimal fashion. Nature, 415, 429433. doi: $10.1038 / 415429$ a

Holmes, N. P. (2007). The law of inverse effectiveness in neurons and behaviour: Multisensory integration versus normal variability. Neuropsychologia, 45, 3340-3345. doi:10.1016/j.neuropsychologia .2007 .05 .025

Johansson, G. (1973). Visual perception of biological motion and a model for its analysis. Perception \& Psychophysics, 14, 201-211.

Keysers, C., Kohler, E., Umiltà, M. A., Nanetti, L., Fogassi, L., \& Gallese, V. (2003). Audiovisual mirror neurons and action recognition. Experimental Brain Research, 153, 628-636. doi:10.1007/ s00221-003-1603-5

Kohler, E., Keysers, C., Umiltà, M. A., Fogassi, L., Gallese, V., \& Rizzolatti, G. (2002). Hearing sounds, understanding actions: Action representation in mirror neurons. Science, 297, 846-848. doi:10.1126/science.1070311

Kozlowski, L. T., \& CutTing, J. E. (1977). Recognizing the sex of a walker from a dynamic point-light display. Perception \& Psychophysics, 21, 575-580.

Loula, F., Prasad, S., Harber, K., \& Shiffrar, M. (2005). Recognizing people from their movement. Journal of Experimental Psychology: Human Perception \& Performance, 31, 210-220. doi:10.1037/0096 $-1523.31 .1 .210$

MendonçA, A. C., \& SAntos, J. A. (2008, July). Auditory footsteps affect visual biological motion orientation detection. Poster presented at the International Multisensory Research Forum (IMRF), Hamburg.
Meredith, M. A., \& Stein, B. E. (1983). Interactions among converging sensory inputs in the superior colliculus. Science, 221, 389-391. doi: $10.1126 /$ science. 6867718

Montepare, J. M., Goldstein, S. B., \& Clausen, A. (1987). The identification of emotions from gait information. Journal of Nonverbal Behavior, 11, 33-42. doi:10.1007/BF00999605

Montepare, J. M., \& ZebrowitZ-McArthur, L. (1988). Impressions of people created by age-related qualities of their gaits. Journal of Personality \& Social Psychology, 55, 547-556.

Pollick, F. E., KaY, J. W., Heim, K., \& Stringer, R. (2005). Gender recognition from point-light walkers. Journal of Experimental Psychology: Human Perception \& Performance, 31, 1247-1265. doi:10.1037/ 0096-1523.31.6.1247

Pollick, F. E., Paterson, H. M., Bruderlin, A., \& Sanford, A. J. (2001). Perceiving affect from arm movement. Cognition, 82, 51-61. doi:10.1016/S0010-0277(01)00147-0

Schouten, B., Moyens, E., Brooks, A., van der Zwan, R., \& VerfaILlie, K. (2008, July). The effect of looming and receding sounds on the in-depth perception of point-light figures. Poster presented at the International Multisensory Research Forum (IMRF), Hamburg.

Schouten, B., Troje, N. F., Brooks, A., van der Zwan, R., \& VerFAILLIE, K. (2010). The facing bias in biological motion perception: Effects of stimulus gender and observer sex. Manuscript submitted for publication.

Troje, N. F. (2002). Decomposing biological motion: A framework for analysis and synthesis of human gait patterns. Journal of Vision, 2, 371-387. doi:10.1167/2.5.2

Vanrie, J., Dekeyser, M., \& Verfaillie, K. (2004). Bistability and biasing effects in the perception of an ambiguous point-light walker. Perception, 33, 547-560. doi:10.1068/p5004

VANRIE, J., \& Verfaillie, K. (2004). Perception of biological motion: A stimulus set of human point-light actions. Behavior Research Methods, Instruments, \& Computers, 36, 625-629.

Vanrie, J., \& Verfaillie, K. (2006). Perceiving depth in point-light actions. Perception \& Psychophysics, 68, 601-612.

Vanrie, J., \& Verfaillie, K. (2008). Perceptual coupling of multiple point-light figures. Visual Cognition, 16, 585-615. doi:10.1080/ 13506280701269334

Verfaillie, K. (2000). Perceiving human locomotion: Priming effects in direction discrimination. Brain \& Cognition, 44, 192-213. doi:10.1006/brcg.2000.1228

Wichmann, F. A., \& Hill, N. J. (2001). The psychometric function: I. Fitting, sampling, and goodness of fit. Perception \& Psychophysics, 63, 1293-1313.

\section{SUPPLEMENTAL MATERIALS}

The demo files described in the Appendix, as well as Movies 1, 2, and 3 (in QuickTime format) illustrating our technique for parametric perspective manipulations, may be downloaded from http://brm .psychonomic-journals.org/content/supplemental. 


\section{APPENDIX}

To support future research, we provide the code of a demo that can serve as an easily modifiable starting point for new experiment code.

Presentation (Neurobehavioral Systems) is a stimulus delivery and experimental control program. If you do not have Presentation installed on your computer to run the code provided here, you can download a free trial of the software, with full functionality for 30 days, from www.neurobs.com/nbs_online/presentation/ trial_procedure.

The demo (available at www.neurobs.com/ex_files/expt_view?id=214 or in the supplement) presents a continuously walking point-light walker in a frontal/back view. The demo starts by showing an orthographic projection of a point-light walker. Repeatedly pressing the left arrow gradually adds more perspective information that the figure is facing away. Pressing the right arrow gradually adds more perspective information that the figure is facing the viewer. Below the figure, the angle of the perspective projection is shown (only for perspective projections).

To view the demo:

1. Download, install, and activate Presentation: Follow the instructions provided at www.neurobs.com/nbs_online/presentation/trial_procedure.

2. Download the following files into the same folder on your computer:

Demo_Schouten_Verfaillie_2009.exp (experiment file containing the settings of the demo)

Demo_Schouten_Verfaillie_2009.sce (scenario file containing the code of the demo)

Walking.txt (coordinate file containing the 3-D coordinates of the point-light figure).

3. Double-click the .exp file in your folder. This opens Presentation with the correct settings.

4. Double-click the .sce file in your folder. This opens the scenario file in the editor window of Presentation.

5. Press F7 to compile and then F5 to run the script.

6. Press the left and right arrows while the demo is running to change the perspective information.

7. Press ESC to stop the demo.

(Manuscript received April 6, 2009;

revision accepted for publication June 20, 2009.) 